\title{
The Impact of COVID-19 Pandemic on Psychiatric Emergency Department Visits - A Descriptive Study
}

\section{Manuel Gonçalves-Pinho ${ }^{1,2,3}$ (D) Pedro Mota $^{3} \cdot$ João Ribeiro $^{3} \cdot$ Silvério Macedo $^{3} \cdot$ Alberto Freitas ${ }^{1,2}$}

Published online: 25 August 2020

(C) Springer Science+Business Media, LLC, part of Springer Nature 2020

\begin{abstract}
The coronavirus disease 2019 pandemic (COVID-19) has an important direct and indirect impact on both physical and mental health. We aim to describe the impact of an emergency state period due to COVID-19 on psychiatric emergency department (ED) visits. We conducted a retrospective observational study analysing all emergency visits occurring at a metropolitan psychiatric ED between March 19th and May 2nd 2019 and 2020 (the beginning/end date of the emergency state which Portugal was under due to COVID-19). Data regarding age, sex, diagnoses, admission date, discharge destiny and status were collected. Diagnoses were classified using the International Classification of Diseases version 9, Clinical Modification (ICD-9-CM). There was a 52.2\% decrease on the number of psychiatric emergency visits during the emergency state period $\left(\mathrm{n}_{2020}=\right.$ 780 vs $\mathrm{n}_{2019}=1633$ episodes). The decrease on psychiatric ED visits was greater in the female sex and in the younger age groups. Episodes with a primary diagnosis of Mood disorders lead the decrease on psychiatric ED visits with $68.3 \%$ less episodes. Schizophrenia and other psychotic disorders was the diagnosis group with the smaller decline (9.8\% decrease). COVID-19 emergency state period had an important impact on the number and characteristics of psychiatric ED visits, reinforcing the great indirect effects of COVID-19 on mental health.
\end{abstract}

Keywords Emergency service $\cdot$ Mental health $\cdot$ Pandemics $\cdot$ Administrative database

\section{Introduction}

The coronavirus disease 2019 pandemic (COVID-19) has had a major impact on the lives of millions of people around the world not only because of its rapid spread and significant

Both Manuel Gonçalves-Pinho, MD and Pedro Mota, MD are First Authors of this manuscript.

Manuel Gonçalves-Pinho

manuelgpinho@med.up.pt

Extended author information available on the last page of the article 
mortality, but also due to the changes in people's daily lives, the devastating impact on economy and the profound reformulation of social structures and health systems. [1]

Since its appearance in China in late December, the SARS-CoV2 virus has spread rapidly to the rest of the world. On March 2, 2020, the first COVID-19 positive patient in Portugal was announced. With the continuous rise of cases reported daily, the first state of emergency in the country's history was declared on March 18, when more than 600 infected people were announced, and was maintained for a total of 45 days, ceased only on May 2, with 25,190 diagnosed cases. [2] Considering that the most well-known and most effective measure facing the spread of this infection is social confinement [3], this decree aimed, among other measures, to establish a quarantine for the general population, seeking restriction of movement of people who had potentially been exposed to this contagious disease. As of this day, services and activities considered non-essential ceased. Simultaneously, Portuguese National Health System had to adjust to ensure response to essential care and the burden imposed by the pandemic. About half a million appointments were canceled and nearly 9,000 surgeries were postponed.

Included in this reorganization, the Portuguese Psychiatry and Mental Health units adapted their assistance to the population, reformulating their wards and limiting appointments and home visits to the most urgent cases; non-urgent outpatient appointments were performed by telephone and other digital health interventions to support continuity of care were embraced. However, these changes did not prevent the temporary closure of several ambulatory facilities for psychiatry patients, as well as the acute day hospitals (one of the main alternatives to inpatient regimen), abruptly interrupting the provision of mental health care to a large number of patients.

Bearing in mind the enormous challenges that the COVID-19 pandemic presents to our society, a profound impact on mental health is expected to occur, especially for those at risk of developing psychiatric illness and those with an already established one. [4, 5] In fact, according to The Portugal National Mental Health Survey, 1st report (Caldas-de-Almeida, 2013) [6], Portugal has a high prevalence of psychiatric illnesses, the second higher prevalence in Europe (following Northern Ireland), and considerably far from other southern European countries, which presented much higher prevalence than the northern ones. It is estimated that in Portugal the lifetime prevalence of at least one psychiatric disorder is $42.7 \%$, surpassed only by the USA (47.4\%), which also contrasts with the estimated prevalence of other European countries, such as Spain and Italy (19.4\% and $18 \cdot 1 \%$, respectively). [7]

Although mental health care is available to all in Portugal, and most of the cost is covered by the National Mental Health System budget, high waiting times for specialized consultation, poor continuity of care at ambulatory level, difficulties in scheduling appointments and barriers in the access of ambulatory care after hospitalization, usually make the psychiatry emergency department (ED) a faster accessible structure for health care. Indeed, according to a study by Alves (2013), the majority of admissions in psychiatric ED were not considered urgent.

The primary aim of this study was to describe and evaluate the impact of COVID-19 pandemic and emergency state on psychiatric emergency department admissions. The secondary aims were to compare clinical and sociodemographic characteristics of patients visiting a psychiatric emergency unit during the 2020 emergency state period and the homologous period of 2019.

\section{Methods}

We conducted a retrospective observational study using an administrative database that collects information of adult emergency department visits at the Centro Hospitalar 
Universitário São João (CHUSJ) in Portugal. CHUSJ is a tertiary hospital center located in the North region of Portugal, with a geographical area covering 27 municipal councils and a global population of 3,000,000 inhabitants. The emergency department includes a psychiatric emergency unit responsible for the psychiatric emergency care provided in the North region of Portugal, representing a true multicentric psychiatric unit, as it incorporates emergency psychiatric care from up to 13 different hospital centers with Psychiatric assistance, open and available 24 hours a day. [8,9]

We selected all emergency visits occurred in the CHUSJ that started or ended in the Psychiatric Unit of the ED between 19th of March and 2nd of May of the year 2019 and 2020. This time interval was selected to match with the beginning and end date of the declared emergency state which Portugal was under due to the COVID-19 pandemic. [10,11] Two subgroups of emergency visits were formed based on the dates described in order to compare the impact of COVID-19 pandemic in the number and characteristics of psychiatric emergency visits. We selected the exact time period (month and day) between 2019 and 2020 in order to prevent the possible effects of seasonality in psychiatric disorders manifestations.

Data regarding age, sex, primary diagnoses, admission date, discharge status and destiny after discharge were collected. All patient identification details were anonymized previously to the data analysis in order to secure patients' personal information. Primary diagnoses at discharge, classified using the International Classification of Diseases version 9, Clinical Modification (ICD-9-CM), were grouped using the Agency for Health Care Quality and Research (AHRQ) Clinical Classifications Software (CCS) for ICD-9-CM.

Descriptive statistical analyses, Independent Sample t tests, Chi-square tests and Pearson Correlation models were performed to assess temporal trends between 2019 and 2020, using IBM SPSS Statistics v.24 for Windows (Armonk, NY: IBM Corp).

\section{Results}

During the 45-days emergency state period (between March 19th and May 2nd of 2020) decreed in Portugal due to the COVID-19 pandemic there were a total of 780 psychiatric emergency visits in the psychiatric emergency unit of CHUSJ.

When comparing with the homologous period of the precedent year, where 1633 episodes were registered, there was a $52.2 \%$ relative decrease on the total number of psychiatric emergency visits between the two periods. (Table 1).

Female patients were responsible for most episodes in both periods, however a higher relative decrease was registered in female patient episodes during the emergency state period $(-56.7 \%$ in female vs. $-45 \cdot 1 \%$ in male patient episodes; $p=0.007)$. (Table 1$)$.

The mean age of patients visiting the psychiatric emergency unit during the emergency state period was $47 \cdot 35$ years, an older mean age when compared to 2019 (45.60 years; $p=$ $0 \cdot 016)$. When analysing relative differences per age group, the older age groups presented lower relative decreases on emergency visits when compared to the younger age groups, with a maximum decrease of $59 \cdot 2 \%$ for the $18-30$ years group and $44 \cdot 1 \%$ to the $>70$ years group. Regarding the Manchester triage level there was a relative increase on the visits associated with the Orange and Green levels episodes and a decrease on the Yellow level episodes.

Around $23.7 \%$ of the psychiatric emergency visits in 2020 ended with the patient being hospitalized in a psychiatric ward $(21 \cdot 8 \%$ in $2019 ; p=0 \cdot 291)$. 
Table 1 Sociodemographic and administrative characterization of the emergency psychiatric visits during an emergency state period (March 19th - May 2nd) in Portugal compared to the homologous period in 2019. (NA Not applicable)

\begin{tabular}{|c|c|c|c|c|c|}
\hline $\begin{array}{l}\text { Year } \\
\text { Emergency visits }(n=)\end{array}$ & $\begin{array}{l}2019 \\
1633\end{array}$ & $\begin{array}{l}2020 \\
780\end{array}$ & $\begin{array}{l}\text { Absolute } \Delta_{2020-2019}(\%) \\
-52 \cdot 2\end{array}$ & $\begin{array}{l}\text { Total } \\
2413\end{array}$ & $\mathrm{p}=$ \\
\hline \multicolumn{6}{|l|}{$\operatorname{Sex}(\% ; n=)$} \\
\hline Male & $38 \cdot 4 ; 627$ & $44 \cdot 1 ; 344$ & $-45 \cdot 1$ & $40 \cdot 2 ; 971$ & \multirow[t]{2}{*}{$\mathrm{p}=0.007$} \\
\hline Female & $61 \cdot 6 ; 1006$ & $55 \cdot 9 ; 436$ & $-56 \cdot 7$ & $59 \cdot 8 ; 1442$ & \\
\hline \multicolumn{6}{|l|}{ Age (years) } \\
\hline Mean (SD) & $45 \cdot 60(16 \cdot 68)$ & $47 \cdot 35(16 \cdot 54)$ & NA & $46 \cdot 17(16 \cdot 65)$ & $\mathrm{p}=0.016$ \\
\hline \multicolumn{6}{|l|}{ Age group $(\% ; n=)$} \\
\hline $18-30$ & $21 \cdot 6 ; 353$ & $18 \cdot 5 ; 144$ & $-59 \cdot 2$ & $20 \cdot 6 ; 497$ & \multirow[t]{5}{*}{$\mathrm{p}=0.078$} \\
\hline $31-50$ & $41 \cdot 6 ; 680$ & $39 \cdot 6 ; 309$ & $-54 \cdot 6$ & $41 \cdot 0 ; 989$ & \\
\hline $51-70$ & $28 \cdot 4 ; 464$ & $32 \cdot 2 ; 251$ & $-45 \cdot 9$ & $29 \cdot 6 ; 715$ & \\
\hline$>70$ & $8 \cdot 3 ; 136$ & $9 \cdot 7 ; 76$ & $-44 \cdot 1$ & $8 \cdot 8 ; 212$ & \\
\hline \multicolumn{3}{|c|}{ Manchester triage level (\%) } & \multicolumn{2}{|l|}{ Relative $\Delta_{2020-2019}(\%)$} & \\
\hline Orange & $12 \cdot 1$ & $17 \cdot 8$ & $+5 \cdot 7$ & $14 \cdot 0$ & $\mathrm{p}=0.001$ \\
\hline Yellow & $80 \cdot 3$ & $74 \cdot 0$ & $-6 \cdot 3$ & $78 \cdot 3$ & \\
\hline Green & $7 \cdot 2$ & $7 \cdot 8$ & $+0 \cdot 6$ & $7 \cdot 4$ & \\
\hline $\begin{array}{l}\text { Admission after emergency } \\
\text { visit }(\% ; n=)\end{array}$ & $21 \cdot 8 ; 356$ & $23 \cdot 7 ; 185$ & $+1 \cdot 9$ & $22 \cdot 4 ; 541$ & $\mathrm{p}=0.291$ \\
\hline
\end{tabular}

By analysing the top 10 primary diagnosis we found that the frequency of all diagnostic categories decreased between the compared time periods. Nevertheless, there were differences in the relative decrease on each category with Schizophrenia and other psychotic disorders having the smaller decrease (-9.8\% compared to the 2019 period) and Mood Disorders registering the higher relative decrease ( $-68 \cdot 3 \%$ compared to the 2019 period). (Table 2).

By detailing the emergency visits according to the primary diagnosis, the female sex was responsible for most episodes in all categories except for Schizophrenia and other psychotic disorders (32.7 and 36.2\% in 2020 and 2019, respectively) and in Attention-deficit conduct and disruptive behavior disorders related episodes (48.2 and 50.6\% in 2020 and 2019, respectively). Differences in sex between time periods were more prominent in the diagnostic categories of Suicide and intentional self-inflicted injury (47.2 and 59.3\% in 2020 and 2019,

Table 2 Primary diagnosis group rank of the emergency psychiatric visits in 2019 and 2020 (March 19th May 2nd) according to AHRQ CCS - Clinical Classification Software

\begin{tabular}{|c|c|c|c|c|c|c|c|}
\hline \multirow[t]{2}{*}{ Primary diagnosis group } & \multicolumn{3}{|l|}{2020} & \multicolumn{3}{|l|}{2019} & \multirow[t]{2}{*}{$\Delta_{2020-2019}(\%)$} \\
\hline & Order & $\mathrm{N}=$ & $\%$ & Order & $\mathrm{N}=$ & $\%$ & \\
\hline Anxiety disorders & 1 & 157 & $20 \cdot 1$ & 1 & 372 & $22 \cdot 8$ & $-57 \cdot 8$ \\
\hline Schizophrenia and other psychotic disorders & 2 & 147 & $18 \cdot 8$ & 3 & 163 & $10 \cdot 0$ & $-9 \cdot 8$ \\
\hline Mood disorders & 3 & 108 & $13 \cdot 8$ & 2 & 341 & $20 \cdot 9$ & $-68 \cdot 3$ \\
\hline $\begin{array}{l}\text { Attention-deficit conduct and disruptive } \\
\text { behavior disorders }\end{array}$ & 4 & 56 & $7 \cdot 2$ & 7 & 79 & $4 \cdot 8$ & $-29 \cdot 1$ \\
\hline Adjustment disorders & 5 & 45 & $5 \cdot 8$ & 4 & 111 & $6 \cdot 8$ & $-59 \cdot 5$ \\
\hline Personality disorders & 6 & 42 & $5 \cdot 4$ & 9 & 64 & $3 \cdot 9$ & $-34 \cdot 4$ \\
\hline Suicide and intentional self-inflicted injury & 7 & 36 & $4 \cdot 6$ & 6 & 81 & $5 \cdot 0$ & $-55 \cdot 6$ \\
\hline Residual codes; unclassified & 8 & 34 & $4 \cdot 4$ & 5 & 83 & $5 \cdot 1$ & $-59 \cdot 0$ \\
\hline Poisoning by other medications and drugs & 9 & 31 & $4 \cdot 0$ & 8 & 71 & $4 \cdot 3$ & $-56 \cdot 3$ \\
\hline $\begin{array}{l}\text { Delirium dementia and amnestic and other } \\
\text { cognitive disorders }\end{array}$ & 10 & 22 & $2 \cdot 8$ & 11 & 56 & $3 \cdot 4$ & $-60 \cdot 7$ \\
\hline
\end{tabular}


respectively) and Delirium, dementia, amnestic and other cognitive disorders ( $45 \cdot 5$ and $60.7 \%$ in 2020 and 2019, respectively).

Throughout the emergency state period there was a constant decrease in the number of ED visits until the end of March $(\mathrm{r}=-0 \cdot 317 ; p=0.292)$ with a constant increase afterwards $(\mathrm{r}=$ $0 \cdot 703 ; p<0 \cdot 001)$. The day with fewer psychiatric ED visits was the day with most diagnosed COVID-19 patients, the 10th of April. (Fig. 1).

The unspecific diagnostic category of "Residual codes" was mainly formed by the ICD-9CM code 780.52 Insomnia, unspecified (51.3\% of all episodes of the Residual code category) and $799 \cdot 3$ Debility, unspecified (31.6\% of all episodes of the Residual code category).

\section{Discussion}

To our knowledge this is the first study describing the effect of COVID-19 pandemic and a period of emergency state in psychiatric ED visits. The primary hypothesis of the present study was that the COVID-19 pandemic would lead to a decrease in the demand for health care at the ED, even considering that Portugal stands out as the major emergency services user per capita within the Organization for Economic Co-operation and Development (OECD). [12] In fact, there was a significant decrease of $52.2 \%$ in the demand for care in the Psychiatry Emergence

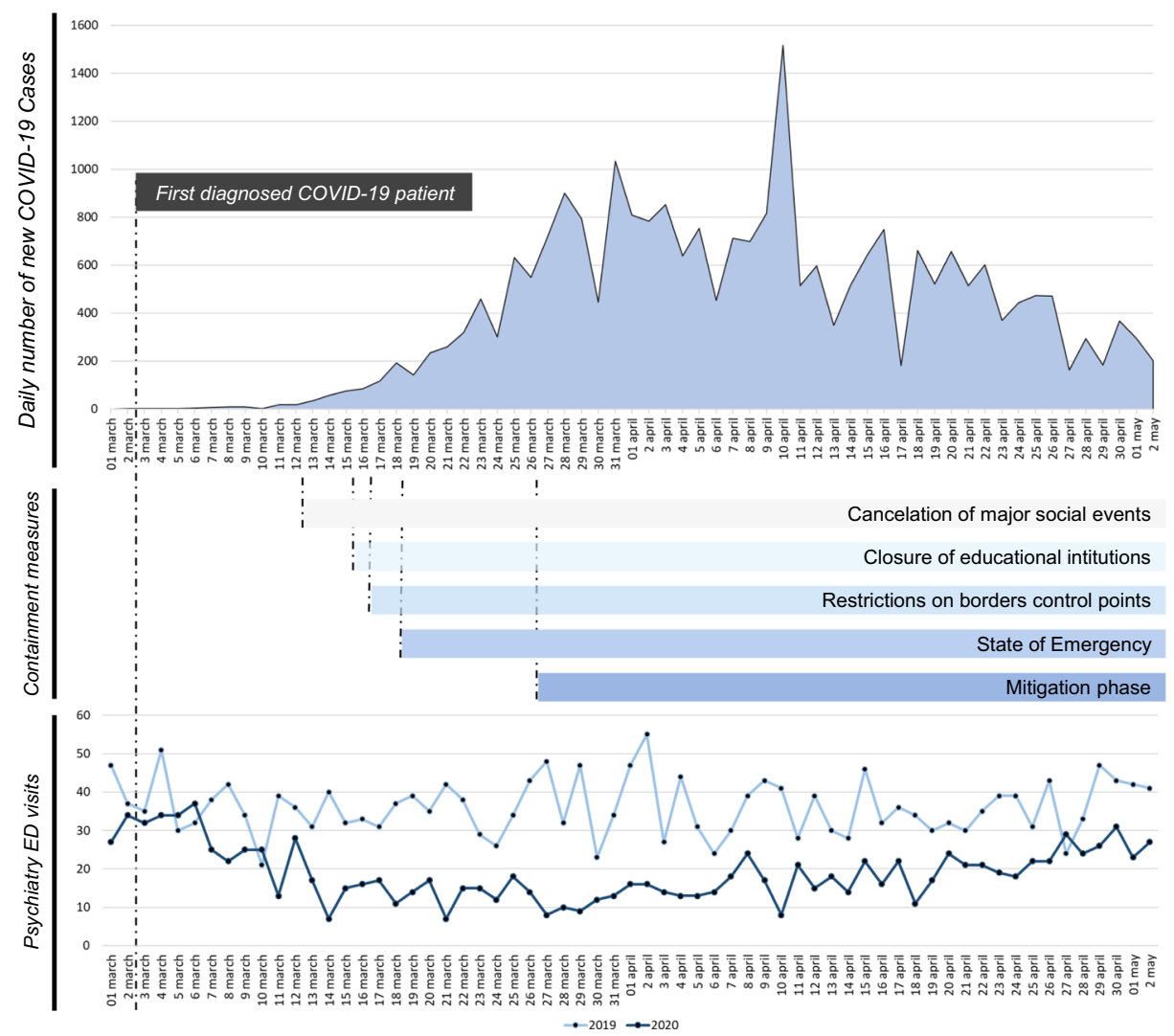

Fig. 1 Distribution of COVID-19 new cases, Psychiatry ED visits and containment measures overtime 
Department in our study population, when compared to the same period of the previous year. These data are in accordance with a recent study developed by the National School of Public Health of the University of Lisbon, which revealed a $45 \%$ drop in visits to emergency departments for all causes in March 2020, when compared to the homologous period in 2019. [13]

The moral conscience of not overloading the services focused on responding to the pandemic, the limitation of displacement and isolation imposed by the quarantine and the fear (and consequent avoidance of risk exposure) of contamination may have been some of the most intuitive reasons that contributed to this decrease, especially among those with nonurgent situations. ED visits are important quality indicators in Mental Healthcare, both in hospital care and primary care.

The analysis of the results shows some agreement with previous studies, such as that of Caldas de Almeida (2010), who reported that the most frequent psychiatric disorders in the Portuguese population are anxiety (16.5\%) and depressive disorders (7.9\%), and that there is a greater prevalence of mental illness in the female patients and in younger age populations. ${ }^{6}$ Interestingly, we found differences in the effect of COVID-19 pandemic and the emergency state period between primary diagnosis categories. Mood disorders arose as the most affected with a decrease on ED visits of $68.3 \%$ contrasting with $9.8 \%$ for Schizophrenia and other psychotic disorders, a difference that may be due to the heterogenous impact of confinement measures, social isolation and accessibility constraints between these group of patients. Previous studies already demonstrated the delayed effect of major military events on the prevalence and incidence of mental disorders, predominantly posttraumatic stress disorder (PTSD) and depressive disorder. [14-17] Although a health pandemic period may not be directly compared to a war period, we can see the differential impact registered on emergency psychiatric care per diagnostic group. (Tables 2 and 3).

Data from this study also show a higher trend of recurrence to the emergency department of older age groups. Pandemic environments of greater social isolation, tend to make older people more vulnerable to symptoms such as anxiety and depression, through a heightened sense of disconnection from society, physical distance and loss of usual social opportunities [18]. Anxiety directly related to the possibility of contracting the virus turns out to be an important factor to consider in this study population. Although the specific routes for anxiety due to the contraction of the disease are still being investigated, it is expected that a contributory characteristic of the fear of contracting COVID-19 involves emotional reactions that are part of a broader behavioral immune system. [19] This pandemic-related anxiety, loneliness and depressive symptoms are also major factors usually related to insomnia.[20]

Unemployment and economic difficulties resulting from this newly installed financial crisis can lead to worsening anxiety and result in greater hopelessness and anger, and present themselves as possible risk factors for suicide. [21] Additionally, in the Northern Hemisphere, suicide rates tend to be higher in late spring and early summer, which coincides with the period of the current evolution of the COVID-19 pandemic, thus requiring increased concern and the need for an adequate assessment. [22] It is also important to consider the seasonal patterns of mood disorders, namely the evidence for a significant seasonal trend for manic episode rates with a peak in spring/summer, also coinciding with the studied period of the pandemic. [23]

Schizophrenia and other psychotic disorders represent an expressive group, confirming a higher prevalence in males and a need for hospitalization for the majority of patients (Table 3 ). Although the absolute number of ED visits did not change significantly during the emergency state period, the relative frequency increased from $10.0 \%$ in 2019 to $18.8 \%$ during the outbreak 


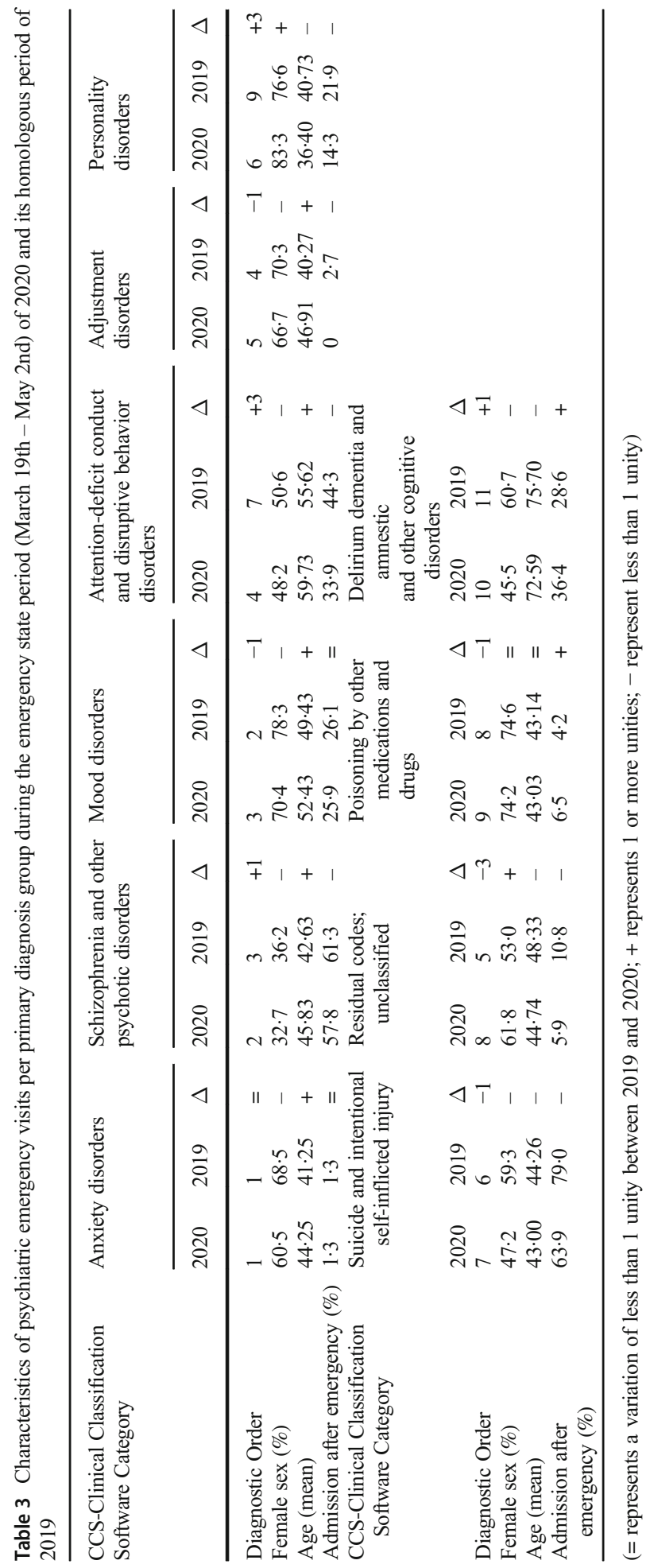


which might be due to the increased need for emergency psychiatric care compared to other group of diagnosis during the COVID-19 pandemic period (Table 2). Psychotic symptoms, such as hallucinations, thought or behavioral disorganization or delusions may lead the patient to dangerous behaviour or thoughts that can represent serious risk, mainly for suicidal ideation or agitation/violent behaviour, which is why psychotic conditions represent serious psychiatric pathologies which often needs urgent intervention in Psychiatry EDs. [24] Although these individuals often lack insight for their condition (and therefore have a lesser willingness to visit emergency departments), intervention by family members or even judicial mechanisms that lead them to these services is often necessary. At the same time, taking into account these characteristics, adherence to the recommended protective measures and compliance with social isolation, may be more difficult for people with schizophrenia, making this group particularly vulnerable to the limitations imposed by the current pandemic situation and more prone to decompensation. It was also hypothesized that COVID-19 infection itself may exacerbate symptoms in people with schizophrenia, as coronaviruses may be associated with psychotic symptoms through an immune-related mechanism. [25]

The Attention-deficit conduct and disruptive behavior disorders and Residual codes; unclassified clusters represent a heterogeneous set of symptoms and pathologies. It includes patients with intellectual disabilities who represent a group whose compliance with isolation measures proves to be problematic, with a tendency to exacerbate psychomotor agitation and aggressive behavior usually aimed at family members or other cohabitants. It is common for these individuals to be integrated into day care centres, closed due to containment measures and possibly explaining the minor decline of psychiatric ED visits during the emergency state period, once the ED was one of the few and more readily available healthcare providers during the pandemic (Tables 2 and 3). The same was true for people living with dementia and other cognitive disorders, a population that is also particularly vulnerable to the pandemic situation, since they are often older people and with other medical comorbidities. These are diseases that, due to their characteristics, can lead them to contradict the confinement measures and family members' recommendations, resulting in periods of greater distress and a tendency to a greater chance of exposure to infection. Also, for those residing in nursing homes, they ended up being deprived of visits from family members, which also contributed to greater psychopathological destabilization and periods of agitation, which often require observation in Psychiatry EDs, with an increase in hospitalization rates among this population in the 2020 period. [26]

The results also demonstrated a higher percentage demand by the Personality disorders group, often represented by a behavioral functioning pattern determined by instability and an intensely disruptive psychological conformation, with an underlying hyper-responsiveness to stress. A global crisis like this and the psychotherapeutic interruption caused by the closure of acute day hospitals, could lead to an increase in psychological destabilization among these patients on a daily basis, often associated with acting-out behaviors, with an increased frequency of drug intoxications and substance consumption.

\section{Limitations}

This study uses an administrative database that collects administrative and clinical information in a major emergency department. As so, the diagnoses are defined in an emergency care setting with all the time and organization constrains associated to it. Psychiatric diagnoses 
require a longitudinal follow-up, and a short emergent evaluation may lead to an incorrect or incomplete diagnosis.

\section{Conclusion}

This is the first study to document the impact of an emergency state period due to the COVID19 pandemic on psychiatric ED visits. We found a general $52 \cdot 2 \%$ decrease on the number of psychiatric ED visits and heterogenous decreases between primary diagnoses groups. Mood disorders were responsible for the most significant decrease (68.3\%) and Schizophrenia and other psychotic disorders for the smaller decrease $(9 \cdot 8 \%)$.

This new pandemic appears to impact mental health through a variety of direct and indirect mechanisms, affecting people with mental disorders differently according to their primary diagnosis.

Acknowledgments We would to like to thank João Viana and Cristina Marujo, MD for their help and support in the extraction and analysis of the data used in this article. We would like to acknowledge the important role that ARSN - Administração Regional de Saúde do Norte and all health professionals working at Centro Hospitalar Universitário São João (CHUSJ) had during the COVID-19 pandemic emergency state period.

Authors' Contributions Author Manuel Gonçalves-Pinho designed the study and participated in all phases of the study. Author Pedro Mota designed the study and participated in all phases of the study. Author João Ribeiro and Silvério Macedo participated in the clinical analyses and writing of the paper. Authors Manuel GonçalvesPinho and Alberto Freitas undertook the statistical analysis, and author Manuel Gonçalves-Pinho wrote the first draft of the manuscript. All authors contributed to and have approved the final manuscript.

Funding Information This work was financed by FEDER - Fundo Europeu de Desenvolvimento Regional funds through the COMPETE 2020 - Operacional Programme for Competitiveness and Internationalisation (POCI), and by Portuguese funds through FCT - Fundação para a Ciência e Tecnologia in the framework of the project POCI-01-0145-FEDER-030766 ("1st.IndiQare - Quality indicators in primary health care: validation and implementation of quality indicators as an assessment and comparison tool").

\section{Compliance with Ethical Standards}

Conflict of Interest The authors declare that they have no conflict of interest.

Research Involving Human Participants and/or Animals The data used was anonymised and all identification variables were not used.

Informed Consent No informed consent was needed once we used an administrative database built for hospital billing with no access to patients identification.

Consent to Participate Not applicable.

Consent for Publication All authors authorize the publication of the manuscript. 


\section{References}

1. Callaway E, Cyranoski D, Mallapaty S, Stoye E, Tollefson J. Coronavirus by the numbers. Nature. 2020;579:482-3.

2. Relatório de Situação da DGS. In: DGS, editor.2020.

3. Lewnard JA, Lo NC. Scientific and ethical basis for social-distancing interventions against COVID-19. Lancet Infect Dis. 2020;20:631-3.

4. Brooks SK, Webster RK, Smith LE, Woodland L, Wessely S, Greenberg N, et al. The psychological impact of quarantine and how to reduce it: rapid review of the evidence. Lancet. 2020;395:912-20.

5. Yao H, Chen J-H, Xu Y-F. Patients with mental health disorders in the COVID-19 epidemic. Lancet Psychiatry. 2020;7(4):e21.

6. Caldas de Almeida JM, Xavier M, Cardoso G, Gonçalves-Pereira M, Gusmão R, Corrêa B, et al. Estudo epidemiológico nacional de saúde mental- $1^{\circ}$ relatório. Lisboa: Faculdade de Ciências Médicas, Universidade Nova de Lisboa; 2013. p. 26-9.

7. Wang PS, Aguilar-Gaxiola S, AlHamzawi AO, Alonso J, Andrade LH, Angermeyer M et al. Treated and untreated prevalence of mental disorder worldwide. Oxford textbook of community mental health 2011:5066.

8. de Pinho Felgueiras JLG. A comparative analysis of psychiatric emergencies in two different emergency department models in Portugal. 2018.

9. Alves AAM. Perfil de utilização da urgência metropolitana de psiquiatria do Porto: um contributo para a análise dos cuidados de Saúde Mental da região: Faculdade de Ciências Médicas; 2013.

10. República Pd. Diário da República n. ${ }^{\circ} 55 / 2020,3^{\circ}$ Suplemento, Série I de 2020-03-18. 2020.

11. República Pd. Diário da República n. ${ }^{\circ} 76 / 2020,1^{\circ}$ Suplemento, Série I de 2020-04-17. 2020.

12. Berchet C. Emergency care services. 2015.

13. Santana R, Sousa JS, Soares P, Lopes S, Boto P, Rocha JV. The Demand for Hospital Emergency Services: Trends during the First Month of COVID-19 Response. Portuguese J Public Health.1-7.

14. Priebe S, Bogic M, Ajdukovic D, Franciskovic T, Galeazzi GM, Kucukalic A, et al. Mental disorders following war in the Balkans: a study in 5 countries. Arch Gen Psychiatry. 2010;67(5):518-28.

15. Karam EG, Mneimneh ZN, Karam AN, Fayyad JA, Nasser SC, Chatterji S, et al. Prevalence and treatment of mental disorders in Lebanon: a national epidemiological survey. Lancet. 2006;367(9515):1000-6.

16. De Jong JT, Komproe IH, Van Ommeren M, El Masri M, Araya M, Khaled N, et al. Lifetime events and posttraumatic stress disorder in 4 postconflict settings. JAMA. 2001;286(5):555-62.

17. De Jong JT, Komproe IH, Van Ommeren M. Common mental disorders in postconflict settings. Lancet. 2003;361(9375):2128-30.

18. Santini ZI, Jose PE, Cornwell EY, Koyanagi A, Nielsen L, Hinrichsen C, et al. Social disconnectedness, perceived isolation, and symptoms of depression and anxiety among older Americans (NSHAP): a longitudinal mediation analysis. Lancet Public Health. 2020;5(1):e62-70.

19. McKay D, Yang H, Elhai J, Asmundson G. Anxiety regarding contracting COVID-19 related to interoceptive anxiety sensations: the moderating role of disgust propensity and sensitivity. J Anxiety Disord. $2020 ; 102233$.

20. Voitsidis P, Gliatas I, Bairachtari V, Papadopoulou K, Papageorgiou G, Parlapani E, et al. Insomnia during the COVID-19 pandemic in a Greek population. Psychiatry Res. 2020;113076.

21. Stuckler D, Basu S, Suhrcke M, Coutts A, McKee M. The public health effect of economic crises and alternative policy responses in Europe: an empirical analysis. Lancet. 2009;374(9686):315-23.

22. Reger MA, Stanley IH, Joiner TE. Suicide mortality and coronavirus disease 2019—a perfect storm? JAMA Psychiatry. 2020.

23. Hochman E, Valevski A, Onn R, Weizman A, Krivoy A. Seasonal pattern of manic episode admissions among bipolar I disorder patients is associated with male gender and presence of psychotic features. J Affect

Disord. 2016;190:123-7.

24. Pandya A, Larkin GL, Randles R, Beautrais AL, Smith RP. Epidemiological trends in psychosis-related emergency department visits in the United States, 1992-2001. Schizophr Res. 2009;110(1-3):28-32.

25. Kozloff N, Mulsant BH, Stergiopoulos V, Voineskos AN. The COVID-19 global pandemic: implications for people with schizophrenia and related disorders. Schizophr Bull. 2020;46:752-7.

26. Wang H, Li T, Barbarino P, Gauthier S, Brodaty H, Molinuevo JL, et al. Dementia care during COVID-19. Lancet. 2020;395(10231):1190-1. 
Publisher's Note Springer Nature remains neutral with regard to jurisdictional claims in published maps and institutional affiliations.

Manuel Gonçalves-Pinho is an Invited Assistant at Faculty of Medicine, University of Porto and a psychiatry resident at Centro Hospitalar do Tâmega e Sousa. He is a member of the research unit CINTESIS (cintesis.eu), belonging to the 2D4H group (Secondary Data for Healthcare Research), and has been involved in projects in several areas of health data science, including health information systems, data quality, intelligent data analysis, performance and quality indicators, coding and audit. He is a Board member of the North Regional Council of the Portuguese Medical Association.

Pedro Mota was born and lives in Portugal and completed his studies in medicine at the University of Porto in 2017. He started specific training in Psychiatry in 2019, showing particular interest in the area of Cultural and Social Psychiatry. As a mental health provider, he is particularly interested in accessing and treating substance use disorders, psychotic disorders and mood disorders. Pedro has also been closely following developments in the therapeutic use of psychedelics for psychiatric illnesses. As his main personal interests, he highlights his interest in the areas of sociology, music, photography and cinema.

João Pedro Ribeiro iis a psychiatrist working at Centro Hospitalar do Tâmega e Sousa in the Inpatient Team in an Acute Psychiatric Ward. He leads the Community Mental Health Team at Centro Hospitalar do Tâmega e Sousa responsible for the mental health proximity care of a geographical region of 500.000 inhabitants.

Silvério Macedo started his studies in Medicine in 2003, at the University of Coimbra, and has been a Psychiatry assistant since 2016. He is particularly interested in the field of Gerontopsychiatry and Neurostimulation.

Alberto Freitas is an Assistant Professor at Faculty of Medicine, University of Porto. In 2007 he achieved the $\mathrm{PhD}$ degree at the School of Economics and Management of the University of Porto, Portugal, with a thesis on the use of data mining techniques for the analysis of hospital inpatient databases. He is member of the research unit CINTESIS (cintesis.eu), with the coordination of the 2D4H group (Secondary Data for Healthcare Research), and has been involved in projects in several areas of health data science, including health information systems, data quality, intelligent data analysis, performance and quality indicators, coding and audit. He has lectured several disciplines on Biostatistics, Hospital Management, and Medical Informatics of pre- and postgraduation courses. He is former director of a master in health informatics (mim.med.up.pt).

\section{Affiliations}

\section{Manuel Gonçalves-Pinho ${ }^{1,2,3} \cdot$ Pedro Mota $^{3} \cdot$ João Ribeiro ${ }^{3} \cdot$ Silvério Macedo $^{3}$ - Alberto Freitas $^{1,2}$}

1 Department of Community Medicine, Information and Health Decision Sciences, Faculty of Medicine, University of Porto, Porto, Portugal

2 Center for Health Technology and Services Research (CINTESIS), Rua Dr. Plácido da Costa, 4200450 Porto, Portugal

3 Department of Psychiatry and Mental Health, Centro Hospitalar do Tâmega e Sousa, Penafiel, Portugal 